\title{
Wall Temperature Measurements Within a High-Power Inductive Plasma Discharge
}

\author{
A. R. Chadwick ${ }^{\circledR}$, T. Janocha, G. Herdrich, B. Dally, and M. Kim
}

\begin{abstract}
Electromagnetic plasma generators (inductive and helicon) are commonly used for planetary entry simulation and increasingly for electric propulsion applications. However, one obstacle in increasing the power and efficiency of these systems is a lack of knowledge regarding the distribution of tube wall temperature. The tube wall temperature acts as a mechanical limit to the thruster/generator operating conditions and is directly related to the distribution of thermal energy within the discharge volume. This paper presents the results of an experimental investigation to determine spatially resolved wall temperatures in a $180 \mathrm{~kW}$ inductive plasma generator. This investigation showed that significant wall heating does not occur until the midpoint of the inductive coil, increasing rapidly to reach peak temperature over a distance $15 \%$ of the total tube. During this rapid heating phase, one-third of the total tube heat flux is produced, assisting in quantifying previously observed mechanical failures due to thermal stress. The peak temperature difference between inner and outer wall surfaces was recorded as $619.1 \mathrm{~K}$, approximately twice that of the previously proposed limit which considered integral rather than spatially resolved values. Using the results of this investigation, a refined estimate of critical wall temperatures for various tube thicknesses has been developed, allowing more targeted cooling systems to be implemented in the future generator designs.
\end{abstract}

Index Terms - Inductive discharge, thermal failure, wall temperature.

\section{INTRODUCTION}

$\mathbf{E}$ LECTRODELESS plasma generator technology has been widely utilized for experimental planetary entry simulation [1]-[3] and the development of advanced, propellantflexible electric propulsion systems [4], [5]. This popularity is attributed to the much purer plasmas produced (devoid of contaminants seen in anode-cathode-based plasma sources) and the flexibility of working gases. This includes chemically

Manuscript received September 16, 2017; revised February 3, 2018; accepted February 8, 2018. Date of publication March 6, 2018; date of current version April 10, 2018. This work was supported in part by the Sir Ross and Sir Keith Smith Foundation and in part by the Deutsche Forschungsgemeinschaft (German Research Foundation) under Project HE 4563/3-1. The review of this paper was arranged by Senior Editor S. J. Gitomer. (Corresponding author: A. R. Chadwick.)

A. R. Chadwick is with the School of Mechanical Engineering, University of Adelaide, Adelaide, SA 5005, Australia (e-mail: ashley.chadwick@dlr.de).

T. Janocha and G. Herdrich are with the Department of Electric Propulsion and Plasma Wind Tunnels, Institute for Space Systems, 70569 Stuttgart, Germany (e-mail: janocha@tesat.de; herdrich@irs.uni-stuttgart.de).

B. Dally is with the Centre for Energy Technology, University of Adelaide, Adelaide, SA 5005, Australia (e-mail: bassam.dally@adelaide.edu.au).

M. Kim is with the Department of Astronautics, University of Southampton, Southampton SO17 1BJ, U.K. (e-mail: m.k.kim@soton.ac.uk).

Color versions of one or more of the figures in this paper are available online at http://ieeexplore.ieee.org.

Digital Object Identifier 10.1109/TPS.2018.2805473 reactive species such as oxygen, which cause severe corrosion in many conventional plasma sources [6], [7]. Despite the separation of plasma and excitation mechanism (coil or antenna), electrodeless plasma sources are still subject to material limitations, particularly in the dielectric material used to form the discharge chamber. While electrodeless devices operating at low powers $(<1-2 \mathrm{~kW})$ and propellant flow rates impart relatively low thermal loads on the discharge chamber, highpower devices $(>20 \mathrm{~kW})$ require dedicated cooling systems to sustain the high-enthalpy plasmas produced [1], [2], [8], [9]. While these cooling systems act uniformly on the outer surface of the discharge tube, tube temperature is a direct result of the discharge translational temperature. Hence, significant nonuniformity in the temperature distribution is observed. An increased understanding of wall temperature distribution can be used to improve future generator designs and decrease heat losses to the discharge chamber material. This is particularly important for high-power inductive electric propulsion, where losses from the discharge volume have a direct impact on achievable spacecraft trajectories. This paper reports on an experimental investigation to obtain spatially resolved wall temperature measurements for a high-power $(180 \mathrm{~kW})$ inductive plasma source. Measurements were performed on the discharge tube inner surface using thermally reactive paint and compared to analytical calculations using the previous integral method to assess the critical tube cooling power. These methods show good agreement and can be used in the development of future inductive plasma sources.

\section{EXPERIMENTAL SETUP}

The plasma source used for these experiments is the Inductive Plasma Generator 7 (IPG7), the seventh generation of IPG developed at the Institute for Space Systems (IRS) [2], [10]. The IPG7, shown in Fig. 1, is capable of a maximum input (anode) power of $180 \mathrm{~kW}$, with seven $6 \mathrm{nF}( \pm 20 \%)$ capacitors used to set the coil driving frequency between 0.5 and 1.5 MHz. For these experiments, a 5.5-turn helical coil (with a total length of $1.5 \mathrm{~m}$ ) and five capacitors were used, resulting in a driving (capacitive) frequency of $586 \mathrm{kHz}$. The cross-sectional diameter of the coil was $12 \mathrm{~mm}$. The generator is attached to a vacuum tank of diameter $2 \mathrm{~m}$ and length $3 \mathrm{~m}$, which is evacuated using the IRS main vacuum facility. This system is able to extract $150000 \mathrm{~m}^{3} / \mathrm{h}$ of air at atmospheric conditions and $250000 \mathrm{~m}^{3} / \mathrm{h}$ at pressures below $10 \mathrm{~Pa}$. The resultant tank pressures are hence less than $1 \mathrm{~Pa}$ without working gas flow and 10-30 Pa during operation. 


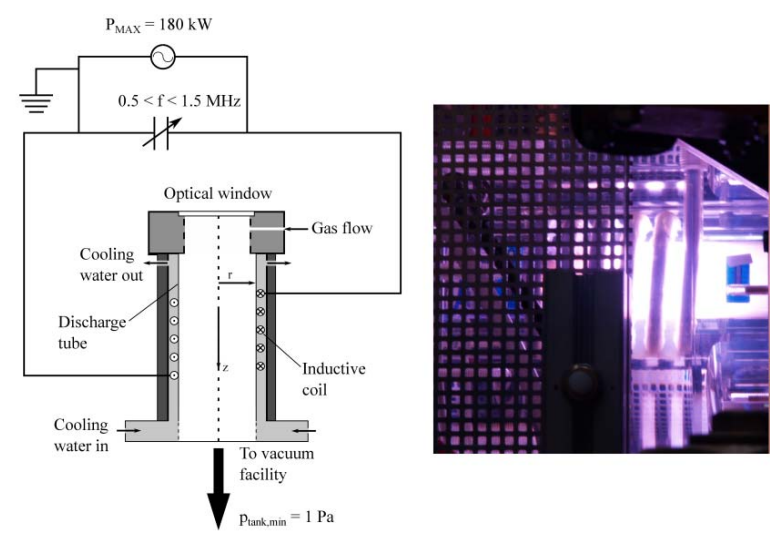

Fig. 1. Left: IPG7 apparatus [11]. Right: during operation with Ar: $\mathrm{N}_{2}$ blend.

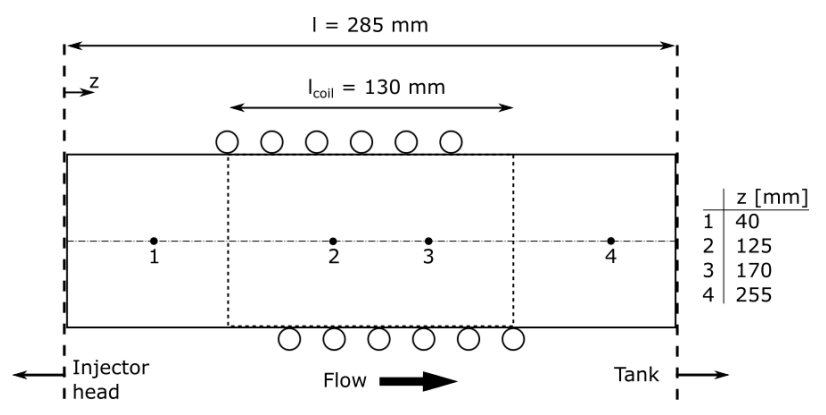

Fig. 2. Thermal paint and adhesive thermometers applied to $3 \mathrm{~mm}$ (wall thickness) tube.

The quartz discharge tube, containing the plasma and acting as a dielectric separation between the inductive coil and working fluid, has a length of $285 \mathrm{~mm}$ and an outer diameter of $90 \mathrm{~mm}( \pm 0.2 \mathrm{~mm})$. Tubes of different wall thicknesses can be fit within this system, having a substantial effect on the discharge behavior [2], [12], [13]. For the experiments conducted in this campaign, a tube of wall thickness $3 \mathrm{~mm}$ $( \pm 0.2 \mathrm{~mm})$ was used.

The internal surface area of this tube was coated with thermally reactive paint suited to high-temperature gas flows [14]. Fig. 2 shows the position of the tube with respect to the inductive coil and the position of adhesive thermometers applied to the tube outer wall.

The paint applied to this tube is designed to change color following exposure to fluid of a particular temperature. Although information on expected transition temperatures is provided with the paint, the in-house calibration is preferable to increase measurement accuracy. This calibration is performed by producing reference paint coloration cells, being smaller quartz tubes treated with the paint and heated to the desired temperature in a Nabertherm HT16/16 furnace. By increasing the temperature gradually in the furnace, the desired temperatures could be reached to $\pm 5 \mathrm{~K}$ accuracy. Given the temperature range of the paint $(<1100 \mathrm{~K})$, $50 \mathrm{~K}$ was selected as an appropriate temperature interval. It should be noted that higher maximum temperature paints (up to $1300 \mathrm{~K}$ ) are available, though with a reduced number of distinct observable transition points. As the transition between
TABLE I

Thermally Reactive Paint Calibration TRANSITION TEMPERATURES

$\begin{array}{cc}\mathrm{T}_{1}[\mathrm{~K}] & <353 \\ \mathrm{~T}_{2}[\mathrm{~K}] & 423 \\ \mathrm{~T}_{3}[\mathrm{~K}] & 473 \\ \mathrm{~T}_{4}[\mathrm{~K}] & 523 \\ \mathrm{~T}_{5}[\mathrm{~K}] & 573 \\ \mathrm{~T}_{6}[\mathrm{~K}] & 623 \\ \mathrm{~T}_{7}[\mathrm{~K}] & 673 \\ \mathrm{~T}_{8}[\mathrm{~K}] & 723 \\ \mathrm{~T}_{9}[\mathrm{~K}] & 773 \\ \mathrm{~T}_{10}[\mathrm{~K}] & 823 \\ \mathrm{~T}_{11}[\mathrm{~K}] & 873 \\ \mathrm{~T}_{12}[\mathrm{~K}] & 923 \\ \mathrm{~T}_{13}[\mathrm{~K}] & 973 \\ \mathrm{~T}_{14}[\mathrm{~K}] & 1023\end{array}$

capacitive (negligible tube heating) and inductive (significant tube heating) is of primary interest, paint was selected to allow sufficient resolution of the heating gradient occurring. The results of this calibration process are listed in Table I. To differentiate between temperature zones following tube testing, these colors were imported into MATLAB and the posttest tube processed in a dedicated script. By measuring the color value of each calibration cell, the error associated with this technique is the sum of the scale used to produce the reference cells and the error in the furnace itself, yielding $\pm 55 \mathrm{~K}$.

As can be seen, the maximum range of the thermal paint $(1023 \mathrm{~K}=750 \mathrm{C})$ limits the discharge conditions which may be investigated using this technique. However, heavy species in contact with the paint are those contained within the discharge thermal boundary layer, not the skin depth region, and hence, inductive discharge can still be investigated using this method. The adhesive thermometers applied to the tube are comprised of nonmetallic materials so as not to interact with the electric or magnetic fields surrounding the thruster. They can hence be placed in close proximity to the coil without compromising discharge coupling. When the tube surface temperature exceeds a given threshold, the organic fluid within the display cells changes its color to indicate the surface temperature in a similar fashion to the thermal paint. The accuracy of the display cell fluid with respect to its indicated temperature is dependent on the temperature range. Combined with the difference between adjacent cell activation, associated errors of the different thermometer classes are listed in Table II. Altered convection over the surface of the tube due to the thermometer's presence may be neglected due to its thin-layer construction. Materials used in the various layers of the thermometer (polyester, Kaladex, acrylic, and paper [15]) are constructed as thin layers in the order of $10^{-5} \mathrm{~m}$, making their heat transfer coefficients negligible in comparison to the quartz surface.

The gas mixture investigated within this paper is one of the combined $\mathrm{Ar}$ and $\mathrm{N}_{2}\left(0.62 \mathrm{Ar}+0.38 \mathrm{~N}_{2}\right)$, with a total 
TABLE II

Adhesive Thermometer Ranges And ACCURACIES [15]

\begin{tabular}{cccc}
\hline Class & $\mathrm{T}_{\min }[\mathrm{K}]$ & $\mathrm{T}_{\max }[\mathrm{K}]$ & Accuracy $[\mathrm{K}]$ \\
\hline $\mathrm{A}$ & 313 & 344 & \pm 7 \\
$\mathrm{~B}$ & 350 & 400 & \pm 7.5 \\
$\mathrm{C}$ & 405 & 455 & \pm 10 \\
$\mathrm{D}$ & 461 & 522 & \pm 12 \\
\hline
\end{tabular}

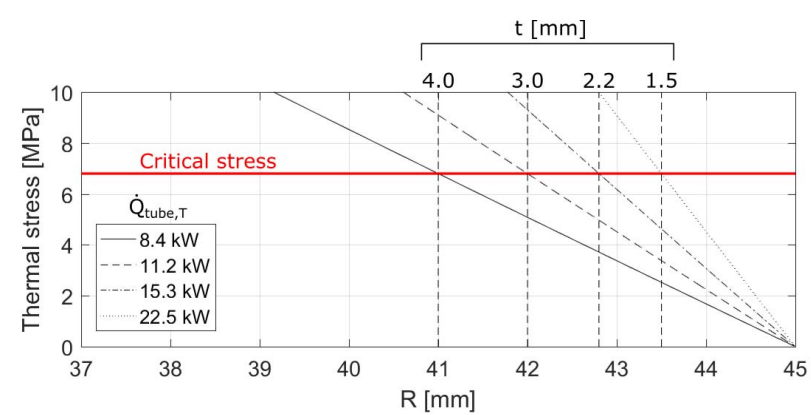

Fig. 3. Tube cooling power at material failure point for various discharge chamber wall thicknesses.

effective mass flow rate of $2.85 \mathrm{~g} / \mathrm{s}$. This mixture was chosen to provide good coupling with the inductive coil (using the low ionization energy of Ar to increase the discharge electron number density) and maintain a high-flow translational temperature (through the higher specific heat capacity of $\mathrm{N}_{2}$ ). While the IPG7 was initially designed for operation with $\mathrm{O}_{2}$, gases containing oxygen were avoided to remove the possibility of additional chemical reactions with the paint. The input (anode) voltage and power to the generator were $5000 \mathrm{~V}$ and $63 \mathrm{~kW}$, respectively, resulting in a plasma jet power of $8.74 \mathrm{~kW}$ measured at the generator outlet using a cavity calorimeter [2]. The low thermal efficiency of this operating condition is due to it lying at the transition point between the capacitive and inductive discharge modes.

\section{Plasma-Wall Interaction}

During IPG7 operation, certain discharge conditions produce sufficient heat flux to exceed material limitations of the water-cooled wall. This has been well documented using integral tube cooling values [2], as shown in Fig. 3. In this paper, as well as the previous studies, tube cooling power $\left(\dot{Q}_{\text {tube }}\right)$ is measured calorimetrically using temperatures of the tube cooling water flow and the water flow rate $\left(\dot{m}_{w}\right)$ to give an integral value of the total thermal load

$$
\dot{Q}_{\text {tube }, T}=c_{p, w} \dot{m}_{w} \Delta T_{w}=\int_{0}^{l} \dot{Q}_{\text {tube }}(l) d l
$$

where $c_{p, w}$ is the isobaric specific heat capacity of the cooling water, $\Delta T_{w}$ is the temperature difference of the cooling water before and after interaction with the discharge tube, and $l$ is the length of the discharge tube. In the case of tube failure, the critical cooling power, $\dot{Q}_{\text {crit }}$, is assumed to be equal to that of heat flux passing through the discharge tube wall (with no additional coupling losses between tube wall and cooling water). Due to the substantial variation in local heat

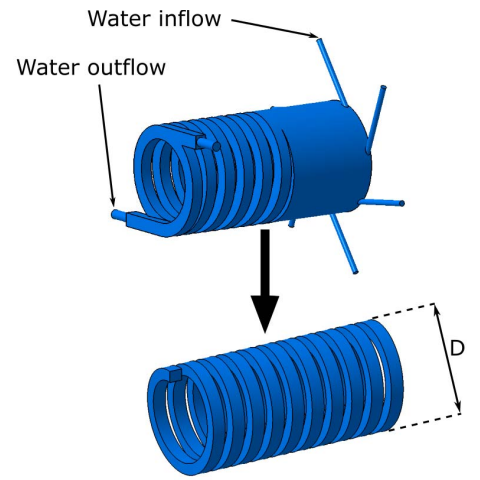

Fig. 4. IPG cooling flow path simplification (adapted from [16]).

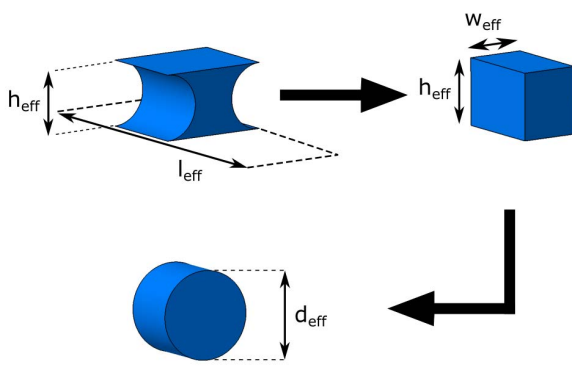

Fig. 5. IPG cooling flow cross-sectional simplification (adapted from [16]).

flux with axial position, mechanical failure often occurs at a distinct axial position such that

$$
\dot{Q}_{\text {tube }}(l)=2 \pi \Delta l \kappa_{\text {tube }} \frac{\Delta T_{\text {wall }}}{\ln \left(\frac{R_{2}}{R}\right)}
$$

where $\Delta l$ is the finite tube section over which the breakage occurs, $\kappa_{\text {tube }}$ is the thermal conductivity of the quartz discharge tube, $\Delta T_{\text {wall }}$ is the temperature difference between the inner and outer tube walls ( $T_{\text {wall, in }}$ and $T_{\text {wall,out }}$, respectively), $R_{2}$ is the outer tube radius, and $R$ is the inner tube wall radius $\left(R_{2}-R=t\right)$. As the tube thickness is increased, the temperature difference required to reach the critical tube stress decreases, causing breakages to occur at lower tube cooling powers. Furthermore, increasing the tube thickness allows less thermal energy to be convected away from the walls by means of the water flow, increasing the inner tube wall temperature whilst decreasing the outer wall temperature. This in turn results in breakages occurring at lower input powers for particular gases.

Equations (1) and (2) are used within this paper to compare experimental measurements with the integral heat flux method used to develop the results in Fig. 3. The same process is also used to determine locally resolved heat flux from the thermal paint method.

In order to assess the tube heat flux analytically, both the water-cooling path around the tube and the path cross section itself must be simplified. This simplification is shown in Figs. 4 and 5. The left-hand image in Fig. 4 represents the flow of cooling water in the laboratory generator. As the maximum tube heating occurs in the vicinity of the coil, the total cooling water volume was simplified to represent 
the helical path caused by the coil geometry. The central image in Fig. 5 is used to calculate the interaction surface area between the tube and cooling water $\left(A_{\text {eff }}=l_{\text {eff }} \times w_{\text {eff }}\right)$, and the right-hand image was used for the flow field calculations (due to the equations' requirement for circular flow geometry [17]). A more detailed explanation of the geometry simplification is available in [16]. Measured values of the tube cooling water temperature $\left(T_{w}\right)$ and total tube heat flux from (1) were used in an iterative process to calculate the tube outer wall temperature at a given experimental condition. The outer wall temperature is calculated as

$$
T_{\text {wall, out }}=\frac{\dot{Q}_{\text {tube }, T}}{A_{\text {eff }} \alpha_{w}}+T_{w}
$$

where $\alpha_{w}$ is the heat transfer coefficient of the cooling water, given as

$$
\alpha_{w}=\frac{\kappa_{w} \mathrm{Nu}}{d_{\mathrm{eff}}}
$$

where $\kappa_{w}$ is the thermal conductivity of the water, $\mathrm{Nu}$ is the flow Nusselt number, and $d_{\text {eff }}$ is the cooling water channel diameter from the circular cross-sectional approximation. This model neglects radiative heat flux from the plasma due to a lack of data on the plasma temperature and emissivity, including the variation of these parameters along the tube axis. Future work on this model would benefit from the inclusion of radiative heat transfer.

Calculating the cooling water Reynolds number (Re), the flow was found to be consistently turbulent. Hence, the Nusselt number for such helical, internally forced convection is given as [17]

$$
\begin{aligned}
\mathrm{Nu} & =\frac{\left(\frac{\zeta}{8}\right) \operatorname{Re} \operatorname{Pr} f_{1}}{1+12.7 \sqrt{\frac{\zeta}{8}}\left(\operatorname{Pr}^{2 / 3}-1\right)} \\
\zeta & =\frac{0.3164}{\operatorname{Re}^{1 / 4}}+0.03\left(\frac{d_{\mathrm{eff}}}{D}\right)^{1 / 2} \\
f_{1} & =\left(\frac{\operatorname{Pr}}{\operatorname{Pr}_{\text {wall }}}\right)^{(7 / 50)} \\
\operatorname{Pr} & =\frac{c_{p, w} \mu_{w}}{\kappa_{w}}
\end{aligned}
$$

where $D$ is the inductive coil outer diameter (and hence cooling channel outer diameter), $\mathrm{Pr}$ and $\mathrm{Pr}_{\text {wall }}$ are the flow Prandtl numbers calculated using the water and outer wall temperatures, respectively, and $\mu_{w}$ is the dynamic viscosity of the cooling water. The term $f_{1}$ is used to account for the wall temperature's influence on the cooling flow, with values of $\mathrm{Pr}_{\text {wall }}$ updated during each iterative cycle until a converged solution to (3) is reached. This result is then combined with (2) to determine either the integral or spatially resolved wall temperature.

\section{EXPERIMENTAL RESULTS}

Images of the painted tube before and after testing are given in Fig. 6, with the tube wall temperatures (inner and outer surfaces) shown in Figs. 7 and 8, respectively. As can be seen from Figs. 7 and 8, no significantly elevated wall temperatures

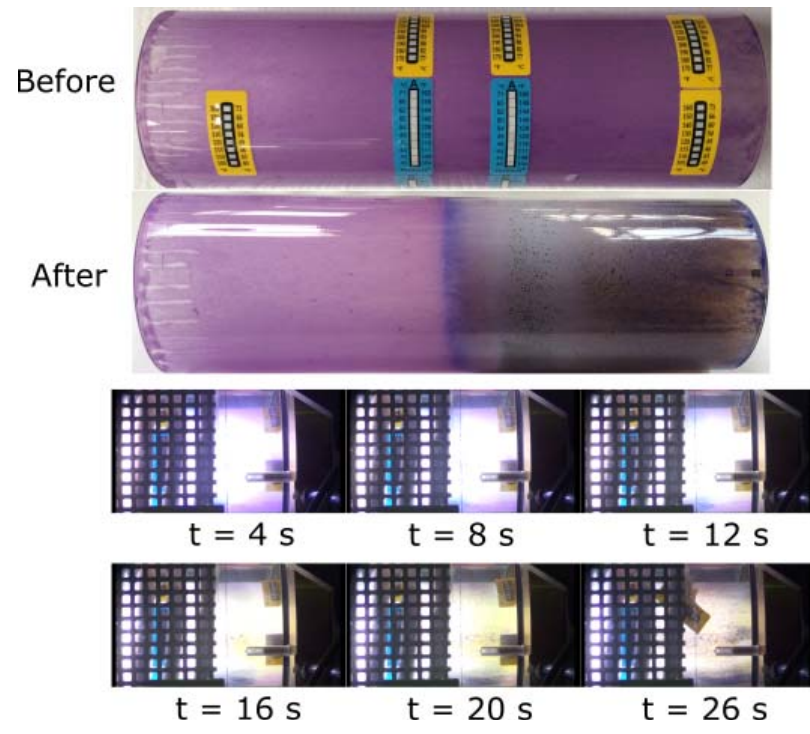

Fig. 6. Thermal paint before and after exposure to the plasma discharge.

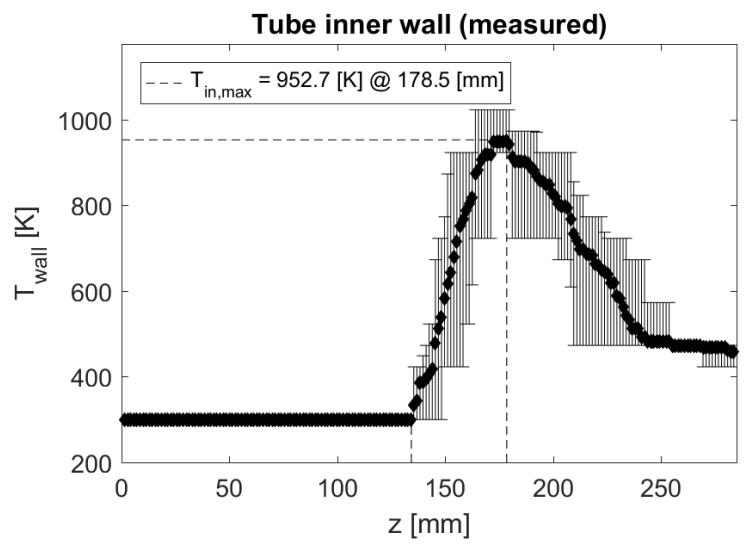

Fig. 7. Measured tube inner wall temperature from thermal paint method.

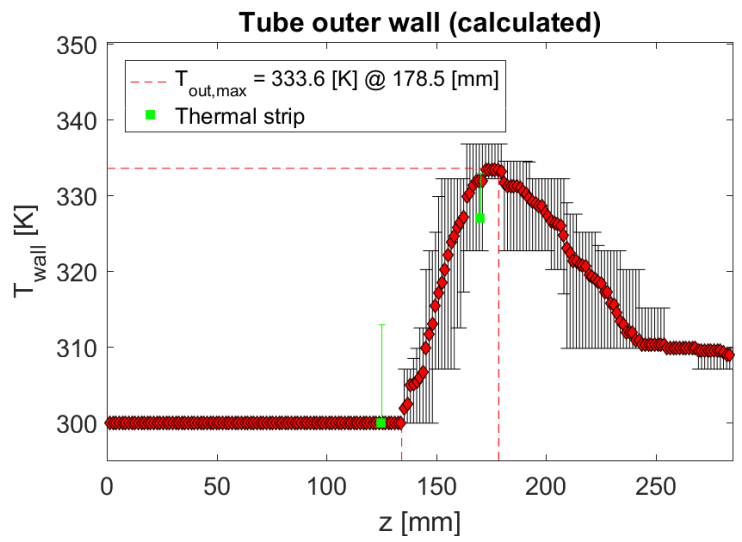

Fig. 8. Calculated tube outer wall temperature from thermal paint method and point measurements from adhesive thermometers.

were recorded until at least the center of the tube, correlating to the midpoint of the inductive coil. While the minimum activation temperature of the paint $(353 \mathrm{~K})$ limits the resolution of this initial transition, the point of maximum recorded temperature, between the final two coil turns (closest to the thruster outlet), agrees well with previously recorded tube 


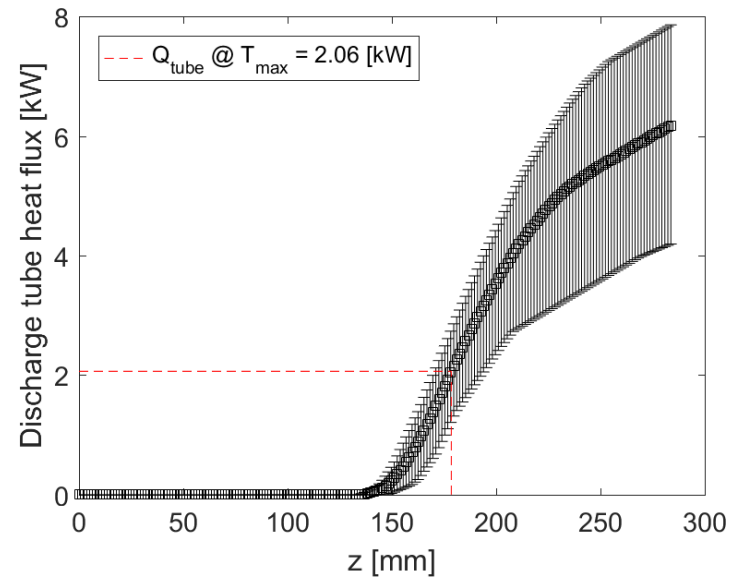

Fig. 9. Distribution of tube wall heat flux over tube axial length.

failures caused by exceeding the integral cooling powers listed in Fig. 3. The paint also reveals steep thermal gradients, with the inner wall temperature increasing by almost a factor of 3 over a small portion of the tube length. Using the modeling method previously discussed [see (2)-(8)], the cumulative tube heat flux with axial position is plotted, as shown in Fig. 9. This plot shows that approximately one-third of the total integral heat flux is incident on the tube over $15 \%$ of its length. This point of rapid heating correlates directly to a local change in the plasma composition and coupling with the inductive coil. As the gas flux used in this system is reasonably high, discharge chamber pressures are two orders of magnitude greater than ambient tank pressures (2500 and $22 \mathrm{~Pa}$ for this experiment, respectively). This high pressure promotes the distribution of thermal energy between heavy particles themselves and between heavy particles and the chamber wall in the form of heat losses to the tube cooling water flow. The pressure difference also biases the flow of energy toward the generator outlet as the diffusion of energetic particles in the upstream direction is limited. As a result, heavy particles moving through the discharge chamber toward the outlet are continually heated (by means of ohmic interaction with the discharge coil) and begin to ionize. This ionization, and the subsequent release of electrons, increases the coupling between the coil and the plasma, and hence, increases the local ohmic heating. In this way, the sharp increase in the tube heat flux can be understood as the point at which significant flow ionization enables a transition between the capacitive and inductive operational modes. This analysis also explains the reduced heat flux gradient toward the end of the coil as proximity to the coil volume decreases and convective heat losses to the tube and vacuum chamber become more significant. It should be noted that the increasingly large error bars with increasing axial position result from a combination of errors in the paint technique and the averaging of measurements at locations around the tube circumference. A total of 10 discrete strip samples were used for the assessment. Comparisons between average temperatures obtained using the paint method and modeled integral cooling method are shown in Table III. The two methods show good agreement in final temperature values, despite a significant (7\%) difference in the respective
TABLE III

Total Tube Heat Flux and Average Wall Temperatures From INTEGRAL AND THERMAL PAINT METHODS

\begin{tabular}{cccc} 
Method & $\dot{Q}_{\text {tube }, T}[\mathrm{~kW}]$ & $\mathrm{T}_{\text {wall, in }}[\mathrm{K}]$ & $\mathrm{T}_{\text {wall }, \text { out }}[\mathrm{K}]$ \\
\hline Integral & 5.78 & 480.34 & 309.08 \\
Thermal paint & 6.20 & 482.99 & 309.73
\end{tabular}

heat flux values. This agreement adds confidence to the results obtained through the thermal paint method, providing new data on the distribution of thermal energy within the discharge tube.

Fig. 8 also shows results from the adhesive thermometers on the tube outer surface. While two of the four thermometers were dislodged during operation (due to interaction with the high-pressure cooling water), those positions at which measurements were obtained show good agreement with the other two methods.

The results obtained during these experiments focus on a particular gas and inductive circuit (coil geometry and operating frequency) in order to obtain information on the wall temperature distribution. This distribution is dependent on many factors and hence different gases, gas flow rates, or inductive coil geometries would produce different peak values and heating gradients. While such variations are of interest to the development of inductive heating systems for particular applications, they lie outside the scope of this paper and will be investigated in subsequent campaigns.

\section{Tube Failure Model}

As shown in Figs. 7 and 8, the maximum recorded temperature difference between the inner and outer wall surfaces was $619.1 \mathrm{~K}$. Although this result matches well with the integral heat flux and thermometer approaches, it is significantly higher than the previously supposed limit of $312 \mathrm{~K}$, determined using [2]

$$
\begin{aligned}
\sigma_{Y} & =\sigma_{\Theta, T}=\alpha_{\text {tube }} E_{\text {tube }} \Delta T_{\text {wall }} \frac{A}{B} \\
A & =2\left(1-v_{\text {tube }}\right) \ln \left(\frac{R_{2}}{R}\right) \\
B & =\left(1-\frac{2 R^{2}}{2 R_{2}^{2}-2 R^{2}} \ln \left(\frac{R_{2}}{R}\right)\right)
\end{aligned}
$$

where $\sigma_{Y}$ is the design yield strength of the quartz tube and $\alpha_{\text {tube }}, E_{\text {tube }}$, and $v_{\text {tube }}$ are the tube coefficients of thermal expansion, Young's modulus, and Poisson's ratio, respectively. This equation, however, assumes heat flux to be distributed over the entire tube length and neglects effects from the compressive force of the cooling water acting on the tube outer surface $\left(7 \times 10^{5} \mathrm{~Pa}\right)$.

Considering both the pressure and temperature components, the hoop stress balance equation is given as

$$
\begin{aligned}
\sigma_{Y} & =\sigma_{\Theta, T}+\sigma_{\Theta, p} \\
\sigma_{Y} & =\alpha_{\text {tube }} E_{\text {tube }} \Delta T_{\text {wall }} \frac{A}{B}+\frac{\left(p_{\text {tube }}-p_{w}\right) R_{2}}{t}
\end{aligned}
$$

where $p_{\text {tube }}$ and $p_{w}$ are the pressures inside the discharge tube and in the tube cooling water, respectively. 


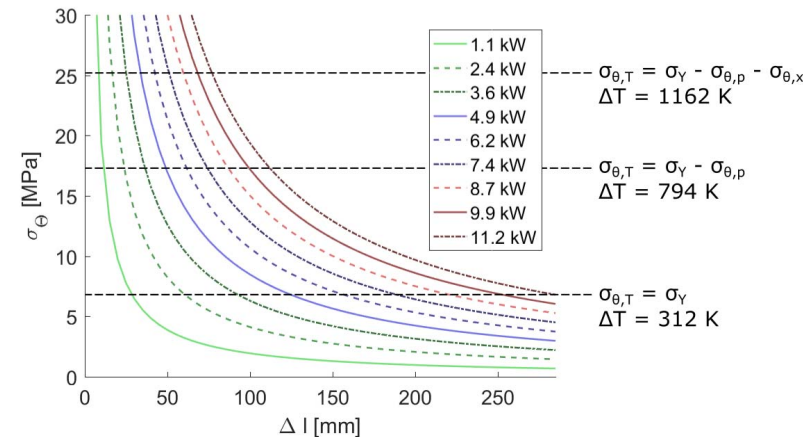

Fig. 10. Tube hoop stress with respect to spatial distribution of thermal power for the $3 \mathrm{~mm}$ tube.

Fig. 10 shows the theoretical critical tube stresses and associated temperatures for the $3 \mathrm{~mm}$ condition investigated, with a limit of $794 \mathrm{~K}$ when considering water-induced tube compression. This value, however, may also under-represent the true temperature difference at tube failure. The maximum measured temperature within this paper, $78 \%$ of the new limit, was obtained at approximately half the critical tube heat flux value. Given the temperature distribution profile shown in Fig. 7 and the consistent material failure at the point of maximum temperature, it is unlikely that this value would increase by just $22 \%$ at the maximum heat flux condition. Due to the increased coupling efficiency associated with higher power operation [2], [11], each increase in input power past this point is expected to significantly increase thermal energy in the discharge and, hence, the tube wall temperature. The true critical temperature is hence proposed lie in the region bounded by the combined thermal and pressure limit (lower bound) and an upper limit that includes other compression stresses caused by components such as the inductive coil. This additional compression stress is termed $\sigma_{\Theta, x}$ and can be determined by solving the full stress balance equation using the calculated maximum input power and measured values of $\Delta T_{\text {wall }}$ and $\dot{Q}_{\text {tube }}$ from experiments

$$
\begin{aligned}
\sigma_{\Theta, x}=\sigma_{Y}-\alpha_{\text {tube }} E_{\text {tube }}\left(\Delta T_{\text {wall, exp }} \frac{\dot{Q}_{\text {crit }}}{\dot{Q}_{\text {tube, exp }}}\right) \frac{A}{B} \\
-\frac{\left(p_{\text {tube }}-p_{w}\right) R_{2}}{t} .
\end{aligned}
$$

This relation results in an upper temperature limit of $1162 \mathrm{~K}$ for the $3 \mathrm{~mm}$ case. It should be noted that this approach assumes minimal broadening of the wall temperature profile due to thermal diffusion, with the locations of peak and initial heating remaining constant. While the assumption of peak heating location is supported by numerous tests of dissimilar gases, the point of initial (significant) tube heating depends on the working fluid composition. This presents a point of investigation for further studies.

Using these upper and lower bound conditions, the failure temperatures and heat flux gradients $(\dot{Q} / \Delta l)$ are plotted in Figs. 11 and 12. As can be seen, the upper limit determined using the modeling analysis and supported by experimental observations show a significantly higher value than the lower,

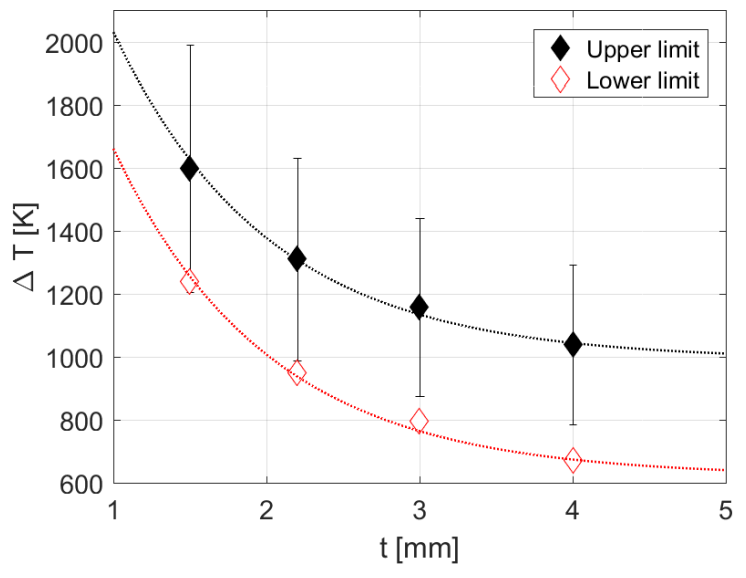

Fig. 11. Upper and lower critical temperatures with respect to tube wall thickness (determined using combined experimental and analytical method).

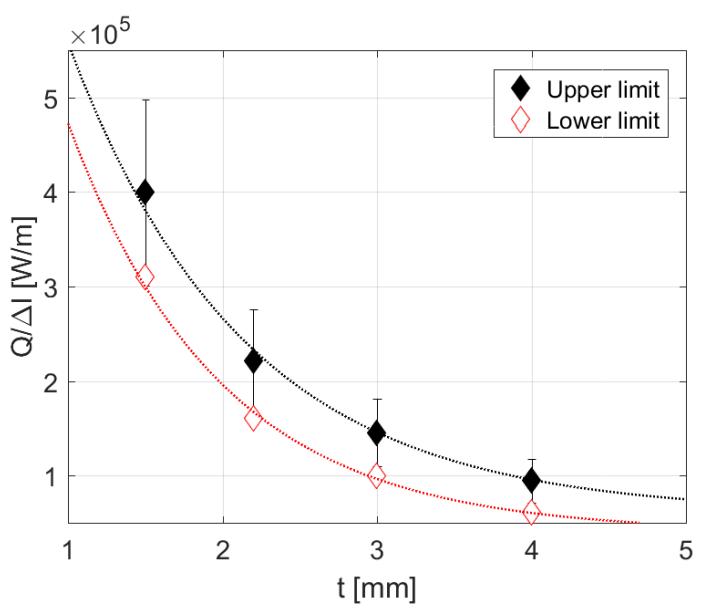

Fig. 12. Upper and lower critical heat flux gradients with respect to tube wall thickness (determined using combined experimental and analytical method).

conservative estimate. As these inductive systems rely on electrothermal interaction to produce their required outlet conditions, the ability to better predict critical failure allows tighter operational tolerances and the ability to significantly increase system efficiency. These values, combined with the distribution of temperature along the tube axis, can be used to improve future generator designs by allowing targeted cooling systems to be produced. Such systems can in turn increase the uniformity of tube wall temperatures and significantly improve discharge coupling.

\section{CONCLUSION}

In this paper, experimental measurements of the distribution of temperature along the discharge tube of a high-power IPG have been shown. These measurements reveal a significant delay in tube heating, with the first significant temperatures correlating with the center of the inductive coil and tube length. The peak temperature difference recorded, $619.1 \mathrm{~K}$, occurred between the final two coil turns, a position which has been extensively recorded as the point of mechanical failure due to thermal loads. At this location, approximately onethird of the total measured heat flux was distributed over $15 \%$ 
of the total tube length. By considering this distribution of temperature and heat flux, an improved estimate of critical temperatures at tube failures has been produced. These two components can be combined to improve the design of future plasma generators for planetary entry and electric propulsion applications, decreasing the quantity of cooling applied to the tube and allowing more targeted systems to be developed.

\section{REFERENCES}

[1] B. Bottin, O. Chazot, M. Carbonaro, D. Van Der Haegen, and S. Paris, "The VKI plasmatron characteristics and performance," The von Kármán Inst. Fluid Dyn., Sint-Genesius-Rode, Belgium, Tech. Rep. ADPO10745, 1999.

[2] G. Herdrich and D. Petkow, "High-enthalpy, water-cooled and thin-walled ICP sources characterization and MHD optimization,' J. Plasma Phys., vol. 74, no. 3, pp. 391-429, 2008, doi: 10.1017/ S0022377807006927.

[3] T. Marynowski, S. Löehle, and S. Fasoulas, "Two-photon absorption laser-induced fluorescence investigation of $\mathrm{CO}_{2}$ plasmas for mars entry," J. Thermophys. Heat Transf., vol. 28, no. 3, pp. 394-400, 2014, doi: 10.2514/1.T4223.

[4] C. Charles, R. W. Boswell, R. Laine, and P. MacLellan, "An experimental investigation of alternative propellants for the helicon double layer thruster,' J. Phys. D: Appl. Phys., vol. 41, p. 175213, Aug. 2008, doi: 10.1088/0022-3727/41/17/175213.

[5] A. Chadwick, B. Dally, M. Kim, and G. Herdrich, "Further development of the TIHTUS test facility at IRS," in Proc. 34th Int. Electr. Propuls. Conf., Jul. 2015, pp. 1-8, paper IEPC-2015-90525.

[6] G. Cifali et al., "Experimental characterization of HET and RIT with atmospheric propellants," in Proc. 32nd Int. Electric Propuls. Conf., Sep. 2011, pp. 1-12, paper IEPC-2011-224.

[7] S. Shiraki et al., "Performance characteristics of low-power arcjet thruster systems with gas generators for water," in Proc. 34th Int. Electr. Propuls. Conf., Jul. 2015, pp. 1-13, paper IEPC-2015-230.
[8] J. Altenberend, G. Chichignoud, and Y. Delannoy, "E-H mode transition of a high-power inductively coupled plasma torch at atmospheric pressure with a metallic confinement tube," Plasma Sources Sci. Technol., vol. 21, no. 4, p. 045011, 2012, doi: 10.1088/0963-0252/21/4/045011.

[9] R. A. Razzak, K. Kondo, Y. Uesugi, N. Ohno, and S. Takamura, "Transition from electrostatic-to-electromagnetic mode in a radio-frequency Ar inductively coupled plasma in atmospheric pressure," J. Appl. Phys., vol. 95, no. 2, pp. 427-433, 2003, doi: 10.1063/1.1635650.

[10] B. Massuti-Ballester, T. Marynowski, and G. Herdrich, "New inductively heated plasma source IPG7," Frontier Appl. Plasma Technol., vol. 6, no. 2, pp. 1-5, 2013.

[11] A. R. Chadwick, G. Herdrich, M. Kim, and B. Dally, "Transient electromagnetic behaviour in inductive oxygen and argon-oxygen plasmas," Plasma Sources Sci. Technol., vol. 25, no. 6, p. 065025, 2016, doi: 10.1088/0963-0252/25/6/065025.

[12] A. R. Chadwick, G. Herdrich, M. K. Kim, B. Dally, and J. Hertel, "Impact of dielectric separation on transition point and accessible flow enthalpy of inductive plasmas," in Proc. Space Propuls., May 2016, pp. $1-10$, paper 3125078 .

[13] A. Nawaz and G. Herdrich, "Impact of plasma tube wall thickness on power coupling in ICP sources," Plasma Sources Sci. Technol., vol. 18, no. 4, p. 045018, 2009, doi: 10.1088/0963-0252/18/4/045018.

[14] TMC Temperature Indicators, Kager $\mathrm{GmbH}$, Dietzenbach, Germany, 2016.

[15] Thermax Irreversible Temperature Recording Strips, Tempil, South Plainfield, NJ, USA, 2016.

[16] T. Janocha, "Wall temperature measurements in an inductively heated plasma generator," (in German), M.S. thesis, Inst. Space Syst., Univ. Stuttgart, Stuttgart, Germany, 2015.

[17] VDI Heat Atlas, VDI-Heat Transfer Technical Handbook, (in German). Heidelberg, Germany: Springer-Verlag, 2010.

Authors' photographs and biographies not available at the time of publication. 